\title{
Cavernoscopic evacuation of aspergilloma: an alternative method of palliation for haemoptysis in high risk patients
}

\author{
R SUBBA RAO, PGD CURZON, MF MUERS, DA WATSON \\ From the Department of Cardiothoracic Surgery and Thoracic Medicine, Killingbeck Hospital, Leeds
}

The reported incidence of pleuropulmonary disease in ankylosing spondylitis ranges from nil to $30 \%$. Rosenow $e t$ $a l^{\prime}$ found a $1.3 \%$ incidence of pleuropulmonary manifestations among 2080 patients suffering from ankylosing spondylitis seen at the Mayo Clinic; five of these 26 patients had aspergillomas. Patients with spondylitis and fibrobullous disease of the lung often develop chronic cavities (usually limited to the upper lobes) and subsequently seem to be particularly prone to develop aspergillomas in these cavities. There is then a risk of massive haemoptysis, which is the main indication for active treatment.

A review of published reports and their own experience prompted Rosenow and colleagues ${ }^{1}$ to comment, "Because of the [high] incidence of [postoperative] complications it seems best to avoid thoracotomy in the patient with ankylosing spondylitis unless it becomes necessary as after major haemoptysis from an aspergilloma." Of the two patients in their series who had pulmonary resection for aspergilloma, one developed a bronchopleural fistula. Among 10 patients with ankylosing spondylitis and aspergillomas discussed by Dewi Davies, ${ }^{2}$ only two of six patients treated by pulmonary resection had a smooth postoperative course; the remaining four developed a bronchopleural fistula. Two of the four non-surgical patients remained free of symptoms while the other two died of haemoptysis.

In this paper we report two patients with ankylosing spondylitis and aspergilloma who were referred to us because of troublesome haemoptysis and were treated by cavernoscopy.

\section{Case reports}

\section{CASE 1}

A man of 45 was diagnosed as having ankylosing spondylitis in 1971. He was referred to a chest physician in September 1981 with repeated attacks of chest infection and massive haemoptysis ( $>300 \mathrm{ml}$ in 24 hours). His haemoglobin concentration was $10 \mathrm{~g} / \mathrm{dl}$. Sputum cultures grew Aspergillus fumigatus and the aspergillus precipitin test was strongly positive. A chest radiograph showed bilateral upper zone fibrosis and features of spinal ankylosis. There was a large cavity containing a fungus ball in the upper zone of the left lung (fig 1a). Respiratory function tests showed severe restrictive lung disease with a forced vital capacity (FVC) of $\mathbf{1 \cdot 8} \mathrm{l}$. In view of the increas-

Address for reprint requests: Mr R Subba Rao, Department of Cardiothoracic Surgery, Killingbeck Hospital, Leeds LS14 6UQ.

Accepted 17 January 1984 ing frequency haemoptysis and the increasing amount of blood lost he was referred for surgery.

Under general anaesthesia a thoracoscope was passed directly into the left upper lobe cavity through the third intercostal space. The fungus ball was visualised, broken up by the biopsy forceps, and removed piecemeal through the thoracoscope until the cavity was empty and its fibrous wall clearly seen. An irrigation catheter was then inserted and left in situ. After operation the cavity was irrigated with $20-30 \mathrm{ml}$ of gentian violet three times daily. This was followed by irrigation with noxithiolin solution for one week. There was no evidence of a communication between the cavity and the bronchial tree. The catheter was removed after 10 days and the patient was discharged home after 12 days.

In the 18 months since the procedure he has remained free of haemoptysis.

Follow up radiographs have revealed no evidence of recurrence of the aspergilloma (fig $1 b$ ). The precipitin test has remained positive but sputum culture has not grown $A$ fumigatus.

\section{CASE 2}

A 24 year old man was diagnosed as having ankylosing spondylitis in 1949 and was treated with radiotherapy. He was found to have bilateral sputum positive pulmonary tuberculosis in 1956 and given treatment. A left apical aspergilloma was first noted on his chest radiograph in 1968 (fig 2a). His main symptoms of cough and haemoptysis were mild until 1981 but both had become worse by June 1981. His sputum culture was positive for $A$ fumigatus and the aspergillus precipitin test was strongly positive. Respiratory function tests showed restrictive lung disease, with an FVC of $2 \cdot 31$. His haemoglobin concentration was $12 \mathrm{~g} / \mathrm{dl}$.

He was referred to us for surgery and underwent cavernoscopic evacuation of the fungus ball, which was hard and needed breaking up into small bits with the tip of a metal suction cannula. The evacuation was undertaken in two sessions, one week apart. As in the previous case, the cavity was irrigated with gentian violet initially, followed by amphotericin B $20 \mathrm{mg}$ daily for seven days. The patient made an uneventful recovery and was discharged 18 days $\varphi$ after the first procedure, free of haemoptysis. He was followed up and remained symptom free for the first six months, but then developed a further but minimal haemoptysis. At this time a chest radiograph showed a recurrence of a fungus ball in the same cavity (fig $2 b$ ). Throughout this period the result of the precipitin test remained strongly positive, although the sputum culture did not grow aspergillus. 


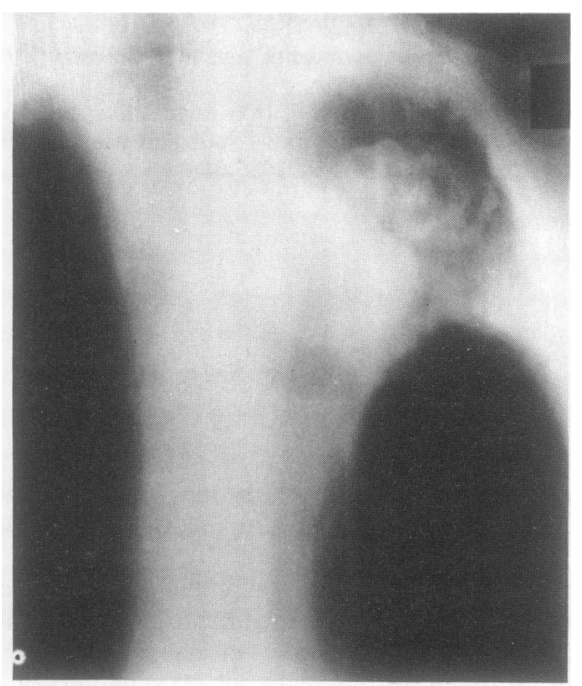

(a)

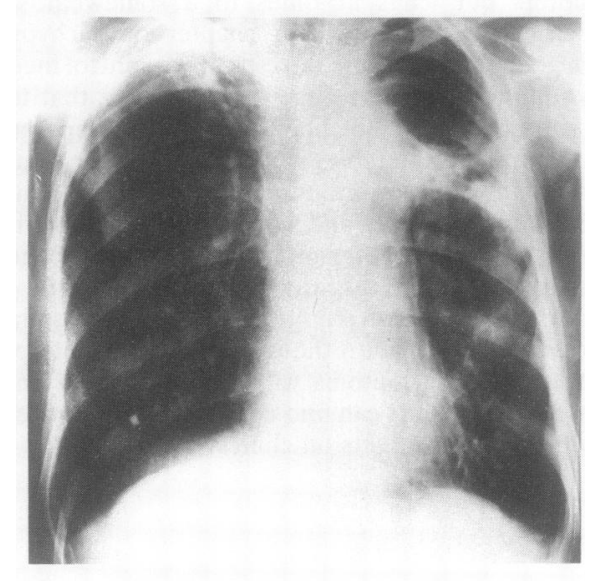

(b)

Fig 1 Case 1: (a) Tomogram, taken before cavernoscopy, of the left upper zone showing a large aspergilloma; (b) chest radiograph, September 1983.

\section{Discussion}

Palliative surgical procedures for the relief of haemoptysis in patients with aspergilloma and severe restrictive lung disease present a difficult therapeutic problem because of the associated morbidity and mortality. The accepted procedure has been to perform cavernostomy and drainage in

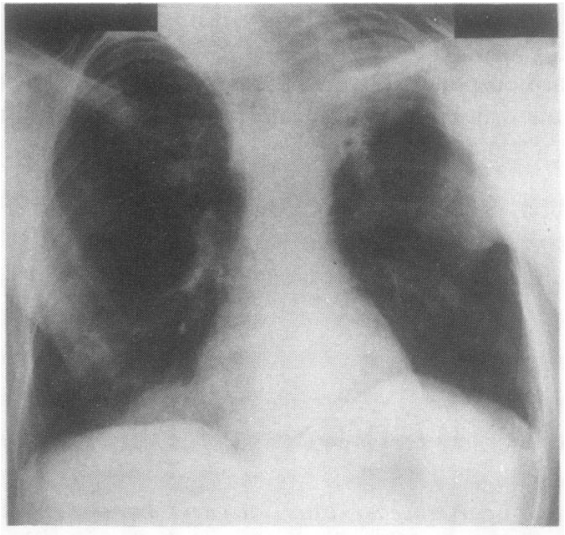

(a)

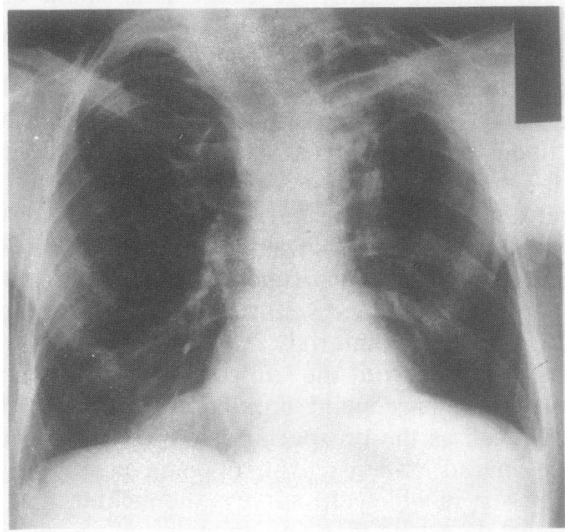

(b)

Fig 2 Case 2: (a) Radiograph, taken before cavernoscopy, showing an aspergilloma in the left upper lobe; (b) chest radiograph, September 1983, showing recurrence of the fungus ball.

such patients, but according to a recent review ${ }^{3}$ this carries a $44 \%$ mortality rate.

The technique of thoracoscopic evacuation of the aspergilloma that we adopted in our two high risk patients was free of complications. The dense adhesions make it easy to pass the thoracoscope into the intrapulmonary cavity without causing pleural dissemination of the fungus or a pneumothorax, and the thick fibrous wall prevents an iatrogenic fistula between the cavity and a bronchus. A higher concentration of antifungal agents can be achieved locally by irrigating the cavity than by oral or intravenous treatment. The second patient has developed a recurrence and we are planning to repeat the procedure if necessary. 
Cavernoscopic evacuation of an intrapulmonary aspergilloma causing appreciable haemoptysis appears to offer a useful alternative approach in high risk patients such as those with ankylosing spondylitis. It appears to be a relatively safe procedure with low morbidity and may be repeated as necessary. It avoids the risks of surgical resection in patients with poor respiratory function and is preferable to open drainage.

Mr Subba Rao is supported by the National Heart Research Fund, Halifax.

\section{Book notices}

Congenital heart disease after surgery: benefits, residua, sequelae. Ed Mary Allen Engle, Joseph K Perloff. (Pp 420; \$40.) Yorke Medical books. 1983.

It is becoming clearer and clearer that truly corrective cardiac surgery hardly exists. Objective assessment of the results of such surgery is essential, both to enable a more realistic picture of the future to be given to the parents of a child whose cardiac lesion is to be repaired and to facilitate improvements in the perioperative management in order to limit undesirable long term sequelae. This timely book covers the field comprehensively, dealing with survival after operation for cyanotic and acyanotic lesions, residual anatomic abnormalities such as ventricular septal defect and aortic stenosis, residual functional anomalies such as arrhythmias and depressed ventricular function, and problems with prosthetic materials. There are also extremely important chapters on the outcome of pregnancy in mothers with repaired and unrepaired congenital heart defects, as well as the prospects for employment and life assurance in our patients. The opening chapter by $\mathrm{Dr}$ Helen Taussig, in which she surveys the incidence of congenital heart defects in man and animals and speculates on the implications for aetiology, is interesting but not germane to the book. The inclusion of three epilogues, albeit by distinguished authors, borders on the maudlin; but in general the book is a mine of information, much of which is not available elsewhere. It will undoubtedly become a standard reference manual.-FJM

\section{References}

' Rosenow EC III, Strimlan CV, Muhm JR, Ferguson RH. Pleuropulmonary manifestations of ankylosing spondylitis. Mayo Clin Proc 1977;52:641-9.

${ }^{2}$ Davies D. Ankylosing spondylitis and lung fibrosis. $Q \mathrm{~J} \mathrm{Med}$ 1972;164:395-417.

${ }^{3}$ Jewkes J, Kay PH, Paneth M, Citron KM. Pulmonary aspergilloma: analysis of prognosis in relation to haemoptysis and survey of treatment. Thorax 1983;38:572-8.

Signs and symptoms in pulmonary medicine. Frederick $\mathrm{L}$, Glauser. (Pp 242; £23·50.) JB Lippincott Company. 1983.

Twelve authors ( 10 from the pulmonary faculty, Medical College of Virginia) contribute to this intended practical guide for medical students, specialised nurses, and respiratory therapists. In each of 21 chapters a brief account of the pathophysiology, recognition, and interpretation of a sign (or symptom) is followed by a table listing the presence or absence and the severity of the sign in 36 diseases. This gives a pleasing uniformity of style in all chapters but leads at times to a somewhat stilted approach. An example is the chapter on tachypnoea, where a good, brief account of respiratory control mechanisms is followed by a five page table showing that tachypnoea can occur in all 36 conditions except sleep apnoea. The numbering in the tables is inconsistent-for example, aspiration lung disease variously appears as 33,36 , and 38 . The accounts of crackles and wheezes and of clubbing are excellent. A whole chapter on asterixis and two pages on sophisticated tests of respiratory muscle strength seem excessive. Nurses, chest physiotherapists, and respiratory therapists wishing to learn the mechanism and interpretation of signs and symptoms will find more than enough here. Medical students can find equally good or more suitable fare in standard texts on clinical methods.-CS 